\title{
Inflationary tensor fluctuations, as viewed by Ashtekar variables and their imaginary friends
}

\author{
Laura Bethke ${ }^{1}$ and João Magueijo ${ }^{1}$ \\ ${ }^{1}$ Theoretical Physics, Blackett Laboratory, Imperial College, London, SW7 2BZ, United Kingdom
}

(Dated:)

\begin{abstract}
We investigate tensor modes in inflationary scenarios from the point of view of Ashtekar variables and their generalizations labelled by Immirzi parameter $\gamma$, which we'll assume imaginary. Being careful to properly define the classical perturbed Hamiltonian by taking several subtleties into account, we reproduce, on-shell, the usual expression found in cosmological perturbation theory. However the quantum Hamiltonian displays significant differences, namely in the vacuum energy and fluctuations of the various modes. We can identify combinations of metric and connection variables representing graviton states, noting that before reality conditions are imposed there are gravitons and anti-gravitons. It turns out that half of these modes have negative energy but after defining the inner product we conclude that they are non-physical and should be selected out. We are left with the usual graviton modes but with a chiral asymmetry in the the vacuum energy and fluctuations. The latter depends on $\gamma$ and on the ordering prescription (namely in the Hamiltonian constraint). Such an effect would leave a distinctive imprint in the polarization of the cosmic microwave background, thus finally engaging quantum gravity in meaningful experimental test.
\end{abstract}

\section{INTRODUCTION}

Inflation [1] is an impressive sociological achievement. Yet, purely on scientific merits, its predictive value has been questioned, with the epithet "theory of anything" being sometimes applied to its vast array of models. This is not entirely fair, considering that, at least within the most "reasonable" models, a number of consistency conditions must be satisfied, notably those relating the spectral index of scalar fluctuations and the amplitude of tensor modes. Should gravitational waves be detected, say via the polarization of the $\mathrm{CMB}$, and should they satisfy these consistency conditions, it would be hard to deny the predictive power of inflation.

And yet, from the point of view of theories of quantum gravity, the inflationary quantum mechanism for generating tensor fluctuations inevitably raises concerns. Strictly speaking, we should be in possession of the full theory of quantum gravity to understand graviton modes even at the perturbative level. One might argue that inflationary fluctuations are protected from the detailed workings of quantum gravity. But delving deeper into the issue reveals that details do matter. A reexamination of inflationary tensor modes from the standpoint of Ashtekar's formalism leads to the prediction of a chiral gravitational wave background [2], unlike in the standard inflationary calculation, based on the second order formalism. This may be a curse or a blessing. From the point of view of quantum gravity it certainly is cause for celebration: it might open up the field to observation, a basic requirement for any truly scientific theory. It is remarkable that it has occasionally been claimed that the problem of quantum gravity has been solved, when no experimental predictions have been made, let alone verified.

In this paper we provide the details behind our recent Letter 2], where the chirality of the perturbative vac- uum of quantum gravity using Ashtekar variables was derived. The Ashtekar formalism constitutes a promising scheme for quantizing the gravitational field $3-6]$. At its core lies the idea that the connection (or its holonomies), rather than the metric, should be the central gravitational variable driving quantization: tools employed to quantize non-perturbative gauge theories, such as the Wilson loop, can then be used leading to great progress. Furthermore the formalism relies on the application of a canonical transformation (dependent on Immirzi parameter $\gamma$ ) upon the Palatini-Kibble spin-connection [6]. For $\gamma= \pm i$ the connection becomes self-dual (SD) or anti-self-dual (ASD), leading to a number of nice mathematical properties and simplifications. The parameter $\gamma$, however, is usually left undefined. It drops out of the classical field equations but is expected to leave a quantum imprint, similar to the theta parameter in QCD.

Specifically, we showed in [2] that the quantum vacuum energy and fluctuations predicted by the theory display a chirality dependent on $\gamma$. For simplicity, we laid out the argument for the extreme cases $\gamma= \pm i$ and then just presented the answer for a general $\gamma$. Here we provide the detailed calculation. The strategy followed in our work is simple: we never stray far from standard cosmological perturbation theory [7, 8]. Well established classical results in Cosmology must obviously have exactly equivalent descriptions in Ashtekar's formalism; if they don't something has gone awry. Furthermore, the Ashtekar quantization procedure should be mapped, in some approximation, onto the usual inflationary calculation of tensor vacuum quantum fluctuations. If experimentally meaningful differences arise, one should cherish them, but also understand their origin. Thus, in this paper we shall present two types of results: classical and quantum. Whereas classically we will just rediscover wellknown results within a different formalism, the quantum mechanics will contain true novelties.

There is a popular belief that cosmological perturba- 
tion theory in Ashtekar's formalism "has all been done before" or "is just an exercise for the student". Nothing could be more far-removed from reality. The exercise is highly nontrivial, particularly the Hamiltonian solution. Even within the second order formalism [9], a Hamiltonian recast of cosmological perturbation theory is far from straightforward (although it has indeed been done before [10]). As for the first order formalism, apart from partial results obtained in the context of loop quantum cosmology [11] (with a different slant, and therefore bypassing important issues), pitifully little has been done.

In Sections II III and IV we will try to patch this ungainly hole. In Section III we solve the Einstein-Cartan equations to find the classical solution for tensor perturbations in a de Sitter Universe in terms of Ashtekar variables. Illuminating insights on duality and helicity are obtained, clearing up some misconceptions found in the literature. Also, the issue of the reality conditions will be clarified (Section III). We then proceed (Section IV) to rediscover our results within the Hamiltonian formalism, essential for canonical quantization. As we will see, one does not recover the results of standard cosmological perturbation theory, unless a number of subtleties are taken into account.

The second part of this paper is concerned with the quantization of this system. In Section $\nabla$ we set up the quantization procedure by obtaining the commutation relations in terms of a mode expansion. Graviton states can then be identified by examining the structure of the Hamiltonian (SectionVI). In general we find twice as many modes as expected. However the reality conditions impose a specific form for the inner product, which renders half of these modes unphysical. These are also the modes which display negative energy and which do not exist classically. If we restrict ourselves to physical modes we therefore recover the standard gravitons; however the vacuum fluctuations and energy turn out to be chiral, establishing the main result in this paper. In Section VII we explain how this chirality may differ when vacuum fluctuations and vacuum energy are contrasted, and how the result depends on the ordering prescription. Finally, in a concluding Section we summarize our results and outline plans for future work.

Throughout this paper we shall use units for which $\hbar=c=1$ and we parameterize the strength of gravity with $l_{P}^{2}=8 \pi G$. We'll be concerned with the real world, so the metric will invariably be Lorentzian; its signature is taken to be -+++ .

\section{CLASSICAL SOLUTION: HELICITY VS DUALITY}

We will first map well-known results pertaining cosmological tensor perturbations into the Ashtekar formalism. A prominent issue is that of relating helicity states (right and left handed) and duality states (self-dual, SD, and anti-self-dual, ASD). Even though the issue was cleared long ago [12], the myth has persisted that these two types of states align: the right handed graviton is $\mathrm{SD}$ and the left handed one ASD. Instead, it should be obvious that this cannot be true: the two types of states can never align because helicity states are real whereas duality states must be complex for a Lorentzian space. Reality conditions therefore relate SD and ASD states; but they can never impose a constraint upon helicity states.

A close analysis reveals that a proper understanding of the relation between these two types of states can only be reached by including positive and negative frequencies into expansions. This simple technical point was made in 12 but missed in much of the subsequent literature (e.g. [13, 14]). It is the cause of much confusion as well as a few paradoxes. For example, the negative energy modes found by Witten [15], associated with perturbations of the Kodama state [16], can never be helicity modes in a Lorentzian space, as explained later in this paper.

\section{A. Conventions and the background solution}

Let the unperturbed Universe be de Sitter space-time foliated using a flat slicing. Using comoving spatial coordinates and conformal time the background metric is therefore:

$$
d s^{2}=a^{2}\left(-d \eta^{2}+d x^{2}+d y^{2}+d z^{2}\right)
$$

with

$$
a=-\frac{1}{H \eta}
$$

where $H^{2}=\Lambda / 3$ and $\eta<0$. The tetrad basis is $e_{\mu}^{I}=a \delta_{\mu}^{I}$ and the non-zero connection forms are $\Gamma_{0}^{i}=H e^{i}$, where $i=1,2,3$ are 3D Lie algebra indices (as usual [5, [] $]$, we use $I$ and $\mu$ for 4D group and space-time indices, respectively). From these we can find the densitized inverse triad, defined as

$$
E_{i}^{a}=\operatorname{det}\left(e_{b}^{j}\right) e_{i}^{a}
$$

and the self-dual connection.

We shall use the following conventions ${ }^{1}$. We dualize with a Levi-Civita symbol derived from $\epsilon_{0123}=1$ (as opposed to $\epsilon^{0123}=1$ ) and define the SD connection so that $\star A^{i}=+i A^{i}$. Furthemore we map anti-symmetric rank2 spatial indices into vectors via $\Gamma^{i j}=\epsilon^{i k j} \Gamma^{k}$ (see [6], pp.127), or equivalently

$$
\Gamma^{i}=-\frac{1}{2} \epsilon^{i j k} \Gamma^{j k}
$$

\footnotetext{
${ }^{1}$ We follow [6], which is just about the only reference where the author deigned to check the consistency of some of the conventions.
} 
(This convention renders the spatial Cartan equation as $\left.D e^{i}=d e^{i}+\epsilon^{i j k} \Gamma^{j} \wedge e^{k}\right)$. Then the SD connection can be defined by:

$$
A^{i}=\Gamma^{i}+\gamma \Gamma^{0 i}
$$

with $\gamma=i$; indeed it is straightforward to check that with our conventions $\star A^{i}=+i A^{i}$. The ASD connection $\left(\star A^{i}=-i A^{i}\right)$ follows from $\gamma=-i$, the Immirzi connection from a general complex $\gamma$, and the Barbero connection from a real $\gamma$.

Therefore, the background (unperturbed) space has densitized inverse triad and self-dual connection given by:

$$
\begin{aligned}
E_{i}^{a} & =a^{2} \delta_{i}^{a} \\
A^{i} & =-\frac{1}{2} \epsilon^{i j k} \Gamma^{j k}+i \Gamma^{0 i}=i H e^{i}
\end{aligned}
$$

where $a=1,2,3$ are spatial indices for the base manifold. It can be easily shown that these satisfy:

$$
B_{i}^{a}+H^{2} E_{i}^{a}=0
$$

where $B_{i}^{a}$ is the $S U(2)$ magnetic field of $A_{i}^{a}$. For a more general $\gamma$ we have

$$
A^{i}=\gamma H e^{i}
$$

leading to

$$
B_{i}^{a}-\gamma^{2} H^{2} E_{i}^{a}=0
$$

\section{B. Perturbation variables}

We want to study tensor perturbations around this background solution. It is standard [8, 17] to write them as:

$$
d s^{2}=a^{2}\left[-d \eta^{2}+\left(\delta_{a b}+h_{a b}\right) d x^{a} d x^{b}\right]
$$

where $h_{a b}$ is a symmetric, TT (transverse and traceless) Cartesian tensor. The associated triad is therefore perturbed as:

$$
e_{a}^{i}=a\left(\delta_{a}^{i}+\frac{1}{2} h_{a}^{i}\right)
$$

so that for the inverse triad and densitized inverse triad we have:

$$
\begin{aligned}
e_{i}^{a} & =\frac{1}{a}\left(\delta_{i}^{a}-\frac{1}{2} h_{i}^{a}\right) \\
E_{i}^{a} & =a^{2}\left(\delta_{i}^{a}-\frac{1}{2} h_{i}^{a}\right),
\end{aligned}
$$

where we have raised and lowered indices in $h_{a b}$ with a Kroenecker- $\delta$ mixing $a$ and $i$ types of indices (i.e. spacetime and algebra indices). More generally, to leading order we may confuse the $a$ and $i$ indices in all perturbation variables, lowering and raising them likewise. Throughout this paper we'll adopt the following convention: we define $\delta e_{a}^{i}$ via the triad

$$
e_{a}^{i}=a \delta_{a}^{i}+\delta e_{a}^{i}
$$

We then raise and lower indices in all tensors with the Kronecker- $\delta$, possibly mixing group and spatial indices. This simplifies the notation and is unambiguous if it's understood that $\delta e$ is originally the perturbation in the triad. With these conventions we therefore have:

$$
\begin{aligned}
e_{i}^{a} & =\frac{1}{a} \delta_{i}^{a}-\frac{1}{a^{2}} \delta e_{i}^{a} \\
E_{i}^{a} & =a^{2} \delta_{i}^{a}-a \delta e_{i}^{a} .
\end{aligned}
$$

We could have adopted any other convention but it turns out that in our case $\delta e_{i j}$ is proportional to the " $v$ " variable beloved by cosmologists [7, 8] (see also Appendix I). For the connection we write:

$$
A_{a}^{i}=\gamma H a \delta_{a}^{i}+\frac{a_{a}^{i}}{a}
$$

As in the usual cosmological treatment we now subject the perturbations to Fourier and polarization expansions; however the Ashtekar formalism presents us with some subtleties. The main issues are:

- If reality conditions are yet to be enforced there must be graviton and anti-graviton modes, so it's essential not to forget the negative frequencies in all expansions, and ensure that they are initially independent of the positive frequencies.

- For a clearer physical picture, it is convenient to use the quantum field theory convention stipulating that for free modes the spatial vector $\mathbf{k}$ points in the direction of propagation for both positive and negative frequencies. This is a simple point, but spurious couplings between $\mathbf{k}$ and $-\mathbf{k}$ modes otherwise come about, e.g. reality conditions constrain gravitons moving in opposite directions [13, 14], which is physically nonsensical.

- If the above is employed, the physical Hamiltonian also should not contain couplings between $\mathbf{k}$ and $-\mathbf{k}$ modes inside the horizon. The presence of such couplings in the formalism [13, 14] merely reflects not having properly identified the direction of propagation (and thus the polarization). As the modes leave the horizon, couplings between $\mathbf{k}$ and $-\mathbf{k}$ may appear, and represent the production of particle pairs by the gravitational field (where the particles in each pair move in opposite directions) [18]. 
Bearing this in mind we thus write:

$$
\begin{aligned}
\delta e_{i j}= & \int \frac{d^{3} k}{(2 \pi)^{\frac{3}{2}}} \sum_{r} \epsilon_{i j}^{r}(\mathbf{k}) \tilde{\Psi}_{e}(\mathbf{k}, \eta) e_{r+}(\mathbf{k}) \\
& +\epsilon_{i j}^{r \star}(\mathbf{k}) \tilde{\Psi}_{e}^{\star}(\mathbf{k}, \eta) e_{r-}^{\dagger}(\mathbf{k}) \\
a_{i j}= & \int \frac{d^{3} k}{(2 \pi)^{\frac{3}{2}}} \sum_{r} \epsilon_{i j}^{r}(\mathbf{k}) \tilde{\Psi}_{a}^{r+}(\mathbf{k}, \eta) a_{r+}(\mathbf{k}) \\
& +\epsilon_{i j}^{r \star}(\mathbf{k}) \tilde{\Psi}_{a}^{r-\star}(\mathbf{k}, \eta) a_{r-}^{\dagger}(\mathbf{k})
\end{aligned}
$$

where, in contrast with previous literature (e.g. [13, 14]), $e_{r p}$ and $a_{r p}$ have two indices: $r= \pm 1$ for right and left helicities, and $p$ for graviton $(p=1)$ and anti-graviton $(p=-1)$ modes. In a frame with direction $i=1$ aligned with $\mathbf{k}$ the polarization tensors are [17]:

$$
\epsilon_{i j}^{(r)}=\frac{1}{\sqrt{2}}\left(\begin{array}{ccc}
0 & 0 & 0 \\
0 & 1 & \pm i \\
0 & \pm i & -1
\end{array}\right) .
$$

The base functions have form

$$
\tilde{\Psi}(\mathbf{k}, \eta)=\Psi(k, \eta) e^{i \mathbf{k} \cdot \mathbf{x}}
$$

and we impose boundary conditions

$$
\Psi(k, \eta) \sim e^{-i k \eta}
$$

when $|k \eta| \gg 1$ for both $+\mathbf{k}$ and $-\mathbf{k}$ directions ${ }^{2}$. Only then does $\mathbf{k}$ point in the direction of propagation, as required. This convention has the essential advantage of identifying the proper physical polarization: until we know in which sense the mode is moving we cannot assign to it a physical polarization. The functions $\Psi_{e}$ and $\Psi_{a}$ can in principle be anything if we allow the amplitudes $e_{r p}$ and $a_{r p}$ to have the necessary time dependence. However, we may choose $\Psi$ functions so that they carry the full time dependence and the $e_{r p}$ and $a_{r p}$ are constant. Hamilton's equations then merely confirm the latter, but $\tilde{\Psi}_{a}^{r p}$ should have both $r$ and $p$ dependence. In these expansions we have already selected the physical degrees of freedom, i.e. the Gauss and diffeomorphism constraints have been implemented to linear order. Whether or not this is good enough will be commented upon later.

\section{Reading off the classical solution}

In order to canonically quantize the theory we need its Hamiltonian formulation. We'll do this in detail in Section IV. but stress that we can read off the answer from cosmological perturbation theory (see Appendix I and also [7, 8]). Indeed the solution presented in Appendix I is equivalent to solving the problem in the second order formalism. Plugging it into the expressions for the

\footnotetext{
${ }^{2}$ We stress that everywhere in this paper $k=|\mathbf{k}|>0$.
}

Ashtekar connection and imposing a torsion-free condition for relating metric and connection is equivalent to solving the Lagrange equations for the Holst action

$$
S=-\frac{1}{2 l_{P}^{2}} \int \Sigma^{I J} \wedge\left(F_{I J}+\frac{1}{\gamma} * F_{I J}\right) .
$$

Bearing this in mind, we can conclude that functions $\Psi_{e}$ must satisfy the same equation as the variable " $v$ " used by cosmologists. We have that $\delta e_{i j}=a h_{i j} / 2$, so $v \sim \delta e_{i j}$. Therefore, in a de Sitter background:

$$
\Psi_{e}^{\prime \prime}+\left(k^{2}-\frac{2}{\eta^{2}}\right) \Psi_{e}=0,
$$

where ' denotes differentiation with respect to conformal time. This has solution:

$$
\Psi_{e}=\frac{e^{-i k \eta}}{2 \sqrt{k}}\left(1-\frac{i}{k \eta}\right)
$$

where the normalization ensures that the amplitudes $e_{r p}$ become annihilation operators upon quantization. In addition, connection and metric are related by the torsion free condition:

$$
T^{I}=d e^{I}+\Gamma_{J}^{I} \wedge e^{J}=0 .
$$

This is solved by

$$
\begin{aligned}
\delta \Gamma_{i}^{0} & =\frac{1}{a} \delta e_{i j}^{\prime} d x^{j} \\
\delta \Gamma_{k i} & =-\frac{2}{a} \partial_{[k} \delta e_{i] j} d x^{j} .
\end{aligned}
$$

With the conventions given above the second of these equations implies:

$$
\delta \Gamma^{i}=\frac{1}{a} \epsilon^{i j k} \partial_{j} \delta e_{k l} d x^{l} .
$$

Therefore the classical solution for the perturbed connection is:

$$
a_{i j}=\epsilon_{i k l} \partial_{k} \delta e_{l j}+\gamma \delta e_{i j}^{\prime}
$$

Inserting decomposition (19) into (30) and using:

$$
\epsilon_{i n l} k_{n} \epsilon_{l j}^{(r)}=-i r k \epsilon_{i j}^{(r)}
$$

we get the expression:

$$
\Psi_{a}^{r p}=\gamma p \Psi_{e}^{\prime}+r k \Psi_{e}
$$

where, we recall, $r= \pm$ for $R / L$ polarizations and $p=$ \pm for graviton positive frequency $(+)$ and anti-graviton negative frequency $(-)$. We have assumed that $a_{r p}=e_{r p}$, that is the amplitudes to be promoted to creation and annihilation operators should be equal for the metric and connection. 
We can now clarify the relation between duality and helicity. They don't align, as claimed. Inside the horizon $(k|\eta| \gg 1)$, equation (22) holds and eq. (32) implies that:

$$
\Psi_{a}^{r p}=(r-i p \gamma) k \Psi_{e} .
$$

Therefore the SD connection $(\gamma=i)$ is made up of the right handed positive frequency of the graviton and the left handed negative frequency of the anti-graviton ${ }^{3}$. The ASD connection contains the remaining degrees of freedom, as shown in the table:

\begin{tabular}{|c|c|c|}
\hline & $r=+[\mathrm{R}]$ & $r=-[\mathrm{L}]$ \\
\hline $\bar{p}=+\quad[G]$ & $\overline{S D}$ & $\overline{\mathrm{ASD}}$ \\
\hline$p=-[\bar{G}]$ & ASD & SD \\
\hline
\end{tabular}

For other values of $\gamma$ this is shared differently, and as the modes leave the horizon $(|k \eta| \sim 1)$ the classification breaks down. Outside the horizon $(|k \eta| \ll 1)$ we have

$$
a_{i j} \approx \gamma H a \delta e_{i j}
$$

Thus in this regime the torsion free condition imposes

$$
\Psi_{a}^{r p}=\gamma p H a \Psi_{e}
$$

(still assuming $a_{r p}=e_{r p}$ ) and therefore all modes and polarizations appear in the connection.

\section{REALITY AND TORSION}

As explained above, reality conditions should never relate different polarizations, or modes $\mathbf{k}$ and $\mathbf{- k}$. If modes propagate along the $\mathbf{k}$ that labels them, then $\mathbf{k}$ and $-\mathbf{k}$ modes, as well as modes with different polarizations, are independent degrees of freedom for a real metric, and therefore can never be constrained by reality conditions. This is ensured by using expansions (19). The reality of the metric $\left(\delta e_{i j}=\delta e_{i j}^{\dagger}\right)$ then implies:

$$
e_{r+}(\mathbf{k})=e_{r-}(\mathbf{k})
$$

and we simply get the constraint that the graviton and anti-graviton are identified, polarization by polarization, mode $\mathbf{k}$ by mode $\mathbf{k}$. This is eminently sensible.

For the connection the situation is somewhat different. Foremost, reality and torsion-free conditions are combined: the connection is allowed to be complex, but only as long as it is consistent with the metric being real, given the torsion-free condition. Thus (for a general imaginary $\gamma)$ :

$$
\begin{aligned}
& \Re A^{i}=\Gamma^{i} \\
& \Im A^{i}=|\gamma| \Gamma^{0 i}
\end{aligned}
$$

\footnotetext{
${ }^{3}$ We note that this result is linked to the conventions spelled out in the paragraph leading to eq. (5). Other conventions are possible, reversing the result in this table and the sign of $\gamma$.
}

makes up the full set of constraints. In the Hamiltonian framework one only imposes the first of these conditions as a constraint, leaving it for the dynamical evolution to discover the second. That is, one only imposes the constraint:

$$
a_{i j}+\bar{a}_{i j}=2 a \delta \Gamma_{i j}=2 \epsilon_{i n k} \partial_{n} \delta e_{k j}
$$

which in terms of expansion (19) becomes:

$$
\begin{aligned}
& \tilde{a}_{r+}(\mathbf{k}, \eta)+\tilde{a}_{r-}(\mathbf{k}, \eta)=2 r k \tilde{e}_{r+}(\mathbf{k}, \eta) \\
& \tilde{a}_{r+}^{\dagger}(\mathbf{k}, \eta)+\tilde{a}_{r-}^{\dagger}(\mathbf{k}, \eta)=2 r k \tilde{e}_{r-}^{\dagger}(\mathbf{k}, \eta),
\end{aligned}
$$

where $\tilde{a}_{r p}=a_{r p} \Psi_{a}^{r p}$ and $\tilde{e}_{r p}=e_{r p} \Psi_{e}$. The evolution should then imply the rest, viz:

$$
a_{i j}-\bar{a}_{i j}=2 \gamma \delta e_{i j}^{\prime}
$$

which in terms of modes translates into:

$$
\tilde{a}_{r+}(\mathbf{k}, \eta)-\tilde{a}_{r-}(\mathbf{k}, \eta)=2 \gamma \tilde{e}_{r+}^{\prime}(\mathbf{k}, \eta) .
$$

\section{THE HAMILTONIAN AND HAMILTON'S EQUATIONS}

We now try to rediscover the results of cosmological perturbation theory, and those derived classically in Section [I, within the Hamiltonian formalism. A proper understanding of the classical Hamiltonian formulation is needed for quantization. As already implied in the Introduction, the exercise is full of surprises. Without proper care taken regarding a number of subtleties, one actually does not recover the results of standard cosmological perturbation theory.

For a general Immirzi parameter the gravitational Hamiltonian is given by:

$$
\begin{aligned}
\mathcal{H}= & \frac{1}{2 l_{P}^{2}} \int d^{3} x N E_{i}^{a} E_{j}^{b}\left[\epsilon_{i j k}\left(F_{a b}^{k}+H^{2} \epsilon_{a b c} E_{k}^{c}\right)\right. \\
& \left.-2\left(1+\gamma^{2}\right) K_{[a}^{i} K_{b]}^{j}\right]
\end{aligned}
$$

where

$$
K_{a}^{i}=\frac{A_{a}^{i}-\Gamma_{a}^{i}(E)}{\gamma}
$$

is the extrinsic curvature of the spatial surfaces (on shell this becomes $K_{a}^{i} \approx \Gamma_{a}^{0 i}$, something that should be discovered by Hamilton's equations). To this volume integral one must add a boundary term [19 21]:

$$
\mathcal{H}_{B T}=-\frac{1}{l_{P}^{2}} \int d \Sigma_{a} N \epsilon_{i j k} E_{i}^{a} E_{j}^{b} A_{b k} .
$$

This term may be zero (e.g. if the manifold has no boundary), but otherwise ignore it at your peril. Its vanishing is often ensured by imposing suitable fall-off conditions [13, 21], but these are blatantly violated by plane 
waves. Therefore in the study of mode solutions (and their Hamiltonian) it is essential to include the boundary term (this matter is usually swept under the carpet by performing an ad-hoc integration by parts in order to obtain the "right result").

Strictly speaking, (44) constitutes the Hamiltonian constraint; the full Hamiltonian is made up of two other constraints, the Gauss constraint:

$$
G_{i}=D_{a} E_{i}^{a}=\partial_{a} E_{i}^{a}+\epsilon_{i j k} A_{a}^{j} E_{k}^{a} \approx 0
$$

and the vector constraint

$$
V_{b}=E_{i}^{a} F_{a b}^{i} \approx 0
$$

(a combination produces the so-called diffeomorphism constraint). These are automatically satisfied to first order by the mode decompositions chosen. A comment on the impact of this for the commutation relations will be made in Section $\mathrm{V}$.

The dynamics is specified by the Hamiltonian together with sympletic structure

$$
\left\{A_{a}^{i}(\mathbf{x}), E_{j}^{b}(\mathbf{y})\right\}=\gamma l_{P}^{2} \delta_{a}^{b} \delta_{j}^{i} \delta(\mathbf{x}-\mathbf{y}) .
$$

We'll now rediscover the usual equations of cosmological perturbation theory in this framework. For clarity we will make our various points restricting ourselves to $\gamma= \pm i$ (as we did in [2]). In the last subsection we'll then list the corresponding results for the general case.

\section{A. Hamilton's equations for $\gamma= \pm i$}

The full Hamilton equations for $\gamma= \pm i$ are:

$$
\begin{aligned}
& A_{a}^{i^{\prime}}=\left\{A_{a}^{i}, \mathcal{H}\right\}=\gamma N \epsilon_{i j k} E_{j}^{b}\left(F_{a b}^{k}+\frac{3}{2} H^{2} \epsilon_{a b c} E_{k}^{c}\right) \\
& E_{i}^{a \prime}=\left\{E_{i}^{a}, \mathcal{H}\right\}=-\gamma \epsilon_{i j k} D_{b}\left(N E_{j}^{a} E_{k}^{b}\right) .
\end{aligned}
$$

It's easy to check that the background solution (6)-(7) solves the Hamiltonian constraint $\mathcal{H} \approx 0$, as well as Hamilton's equations. In performing this exercise note that using conformal time, the lapse density is $N=1 / a^{2}$. This is a simple test on consistency of conventions. The background solution also trivially satisfies the Gauss and vector constraints (47)-(48).

If we perturb these equations via (17) and (18) we find:

$$
\begin{aligned}
a_{i j}^{\prime} & =2 \gamma H^{2} a^{2} \delta e_{i j}-\gamma \epsilon_{i n m} \partial_{n} a_{m j} \\
\delta e_{i j}^{\prime} & =-\gamma\left(a_{i j}-\epsilon_{i n m} \partial_{n} \delta e_{m j}\right) .
\end{aligned}
$$

The Hamilton equation for $\delta e_{i j}$ is simply (30) for $\gamma=$ $\pm i$, i.e. a statement that on-shell the connection is the torsion-free SD or ASD connection (or alternatively, a confirmation that the extrinsic curvature is $\delta K_{i j}=e_{i j}^{\prime}$; cf. Eqn (45)). Combining equations (51) and (52) we obtain the familiar second order equation for $\delta e_{i j}$ :

$$
\delta e_{i j}^{\prime \prime}-\left(\partial^{2}+\frac{2}{\eta^{2}}\right) \delta e_{i j}=0
$$

equivalent to (133) in Appendix I. This shows that classically, the standard formalism of cosmological perturbation theory and the Hamiltonian Ashtekar framework are equivalent.

\section{B. The perturbative status of the Hamiltonian constraint}

As we've seen it's easy to find the perturbation equations by perturbing the full Hamilton's equations. However, locating the perturbed Hamiltonian within the full theory is more subtle. This is to be expected from the fact that the usual Hamiltonian constraint $\mathcal{H} \approx 0$ cannot apply to the perturbative Hamiltonian, since gravitons do have dynamics.

The first order Hamiltonian is trivially zero (once the other constraints are used). The second order Hamiltonian is made of two terms:

$$
{ }^{2} \mathcal{H}={ }_{1}^{2} \mathcal{H}+{ }_{2}^{2} \mathcal{H},
$$

where ${ }_{1}^{2} \mathcal{H}$ contains products of first order perturbations and ${ }_{2}^{2} \mathcal{H}$ contains second order perturbations in the triad and connection. Only the total must vanish on shell. The first term provides a candidate for the Hamiltonian to be identified with that of the second quantized QFT. The second contains the backreaction or compensation resulting from the non-linearity of the gravitational field, ensuring that the Hamiltonian constraint is satisfied, whilst a strictly positive ${ }_{1}^{2} \mathcal{H}$ remains possible. We'll concentrate on term ${ }_{1}^{2} \mathcal{H}$ and so ignore the Hamiltonian constraint to second order (which would only provide us with information on the backreaction term).

Before we proceed with the algebra we need to stress a crucial point: In the formulation we are using, the Hamiltonian is not real. Of course, after the constraints are imposed the Hamiltonian becomes weakly zero and therefore real. However, off-shell we have to deal with an intrinsically complex Hamiltonian. Furthermore this distinctive feature propagates into perturbation theory. The Hamiltonian constraint doesn't apply to the piece of the Hamiltonian, ${ }_{1}^{2} \mathcal{H}$, which provides the raw material for the second quantized theory living inside the full non-perturbative theory. Therefore ${ }_{1}^{2} \mathcal{H}$ is complex, and we have to live with it. Some of the novelties to be derived in this paper trace their origin directly to this fact. As we will explain later, however, the Hamiltonian is still Hermitian with respect to an inner product still to be defined (and the two matters, complexity and Hermiticity, should not be confused).

By expanding the Hamiltonian to second order we find:

$$
\begin{aligned}
{ }_{1}^{2} \mathcal{H}= & \frac{1}{2 l_{P}^{2}} \int d^{3} x\left[-a_{i j} a_{i j}+2 \epsilon_{i j k} \delta e_{l i} \partial_{j} a_{k l}\right. \\
& \left.-2 \gamma H a \delta e_{i j} a_{i j}-2 H^{2} a^{2} \delta e_{i j} \delta e_{i j}\right] .
\end{aligned}
$$

Note that in obtaining (44) from the usual ADM action (by means of an extension of the phase space and a 
canonical transformation), a number of algebraic manipulations are needed in which the Gauss (or "rotational") constraint and the torsion free condition are used (see [6]; chapters 1 and 4). However, when evaluating the Hamiltonian to second order, ${ }_{1}^{2} \mathcal{H}$, we have to bear in mind that expansions (19) only solve the Gauss constraint to linear order, so that to second order

$$
{ }_{1}^{2} G_{i}=-\epsilon_{i j k} a_{a}^{j} \delta e_{k}^{a} \neq 0 .
$$

Thus, terms linear in ${ }_{1}^{2} G_{i}$, multiplied by zero order variables, will appear in ${ }_{1}^{2} \mathcal{H}$. Likewise, with solution (29), the torsion $T^{a}=d e^{a}+\Gamma_{b}^{a} \wedge e^{b}$ only vanishes to first order, and has a non-zero contribution quadratic in first order perturbation variables, i.e. ${ }_{1}^{2} T^{a} \neq 0$. This affects several manipulations leading to ${ }_{1}^{2} \mathcal{H}$, and even the definition of the covariant derivative with respect to the Ashtekar connection (note that the Gauss constraint is initially a "rotational" constraint, $G_{i}=\epsilon_{i j k} K_{a}^{j} E_{k}^{a}$, and not a proper Gauss law; see [6], pp.124). It can be checked that all extra terms in ${ }_{1}^{2} \mathcal{H}$ resulting from these considerations form a full divergence irrelevant for the purpose of this paper.

\section{Two further subtleties}

It may seem that we have identified the portion of the full Hamiltonian to be associated with the perturbative Hamiltonian to second order, but this is not the case. It's easy to check that (55) does not reduce to the expected expression (132) in Appendix I on-shell, i.e. using (30). This is due to two reasons.

Firstly, one must add the corresponding boundary term (46) at the same order and level in perturbation theory (i.e. second order terms quadratic in first order variables). This is:

$$
{ }_{1}^{2} \mathcal{H}_{B T}=\frac{1}{l_{P}^{2}} \int d \Sigma_{i} \epsilon_{i j k} \delta e_{l j} a_{l k}
$$

which should be brought down to the interior of the region in the form of a divergence. When this is done we obtain:

$$
\begin{aligned}
\mathcal{H}_{e f f}= & \frac{1}{2 l_{P}^{2}} \int d^{3} x\left[-a_{i j} a_{i j}-2 \epsilon_{i j k}\left(\partial_{j} \delta e_{l i}\right) a_{k l}\right. \\
& \left.-2 \gamma H a \delta e_{i j} a_{i j}-2 H^{2} a^{2} \delta e_{i j} \delta e_{i j}\right]
\end{aligned}
$$

and if we can ignore terms in $H$ it's easy to check that classically (on-shell) this is nothing but the usual expression for the stress-energy tensor of gravitational waves, with a kinetic and a gradient term as usual. However, if terms in $H$ cannot be neglected this is still not Eq. (132).

In order to understand why, it's well worth looking at "perturbative" expressions

$$
\begin{aligned}
& A_{a}^{i}=\gamma H a \delta_{a}^{i}+\frac{a_{a}^{i}}{a} \\
& E_{i}^{a}=a^{2} \delta_{i}^{a}-a \delta e_{i}^{a}
\end{aligned}
$$

in a different way: they represent a canonical transformation. Above all we have traded canonical variables $\left(A_{a}^{i}, E_{j}^{b}\right)$ with variables $\left(a_{a}^{i}, \delta e_{j}^{b}\right)$; and only then, in what might be properly called perturbation theory, have we assumed the latter to be small, so that a truncation scheme can be set up. However the transformation can always be carried out, even when $\left(a_{a}^{i}, \delta e_{j}^{b}\right)$ are not small and no truncations are applied.

This way of thinking has several advantages. Firstly it permits a rigorous derivation of the sympletic structure for the new variables:

$$
\left\{a_{a}^{i}(\mathbf{x}), \delta e_{j}^{b}(\mathbf{y})\right\}=-\gamma l_{P}^{2} \delta_{a}^{b} \delta_{j}^{i} \delta(\mathbf{x}-\mathbf{y})
$$

(where the minus sign appears because $\delta e_{i j}$ is the perturbation in the triad, not the densitized inverse triad) ${ }^{4}$. We'll use these Poisson brackets to define the quantum theory in the next Section. Contrary to a common myth we are not "freezing" the background, and allowing the perturbations to "quantum fluctuate" 5 . We are simply replacing a canonical pair by another, which is particularly suited to our problem, since its classical variables can be assumed to be small.

Furthermore the matter is far from pedantic if $a$ is not a constant, because the transformation is then time dependent. Therefore the new Hamiltonian (sometimes represented by $K$; see [22]) is not simply the old Hamiltonian written in terms of the perturbations $\left(a_{a}^{i}, \delta e_{j}^{b}\right)$ (and then possibly truncated). Instead it must be replaced by:

$$
\mathcal{K}=\mathcal{H}+\frac{\partial F}{\partial \eta}
$$

where $F$ is the generating function of the canonical transformation, if we want to obtain an equivalent pair of Hamilton's equations. It can be easily checked that if we take the perturbed (old) Hamiltonian (58) and work out Hamilton's equations following from (61) we'd be presented with a result inconsistent with (51) and (52), obtained by evaluating Hamilton's equations and then perturbing. This is prevented by using

$$
\frac{\partial F}{\partial \eta}=\frac{\gamma}{l_{P}^{2}} \int d^{3} x H a \delta e_{i j} a_{i j} .
$$

In Appendix II this generating functional is derived for general values of $\gamma$.

Therefore, we obtain as the Hamiltonian for the new variables:

$$
\begin{aligned}
\mathcal{H}_{e f f}= & \frac{1}{2 l_{P}^{2}} \int d^{3} x\left[-a_{i j} a_{i j}-2 \epsilon_{i j k}\left(\partial_{j} \delta e_{l i}\right) a_{k l}\right. \\
& \left.-2 H^{2} a^{2} \delta e_{i j} \delta e_{i j}\right]
\end{aligned}
$$

\footnotetext{
4 According to some conventions we should endow the new variables $\left(a_{a}^{i}, \delta e_{j}^{b}\right)$ with the same brackets as $\left(A_{a}^{i}, E_{j}^{b}\right)$. A minus sign would then appear in front of the perturbative Hamiltonian.

5 A related myth is that there is no Poisson bracket between zero and first order variables because they "fluctuate independently".
} 
This Hamiltonian should be identified with the Hamiltonian of the second quantized, effective quantum field theory representing the theory perturbatively. And indeed, "on-shell", i.e. using (30), this does finally reduce to Eq. (132).

\section{A general $\gamma$}

The various points made in the previous subsections, for $\gamma= \pm i$, remain valid in the general case, but the algebra is far more complex. Here we list the corresponding results. Hamilton's equations for a general $\gamma$ take the form:

$$
\begin{aligned}
A_{a}^{i^{\prime}}= & \gamma N \epsilon_{i j k} E_{j}^{b}\left(F_{a b}^{k}+\frac{3}{2} H^{2} \epsilon_{a b c} E_{k}^{c}\right) \\
- & \gamma\left(1+\gamma^{2}\right) N E_{j}^{b}\left(K_{b}^{j} K_{a}^{i}-K_{a}^{j} K_{b}^{i}\right) \\
- & \frac{1+\gamma^{2}}{l_{P}^{2}} \int d^{3} y N E_{j}^{b} E_{k}^{c}\left\{A_{a}^{i}(x), \Gamma_{[b}^{j} \Gamma_{c]}^{k}\right\} \\
E_{i}^{a \prime}= & -\gamma \epsilon_{i j k} D_{b}\left(N E_{j}^{a} E_{k}^{b}\right) \\
& +\left(1+\gamma^{2}\right) N\left(E_{i}^{a} E_{j}^{b}-E_{j}^{a} E_{i}^{b}\right) K_{b}^{j}
\end{aligned}
$$

As far as we are aware there is no simple expression for the Poisson bracket $\{A, \Gamma(E)\}$, and so we left the last term of the first equation unexpanded ${ }^{6}$. It is again easy to prove that the zero order solution (6)-(7) satisfies these equations as well as all the constraints. It is also straightforward to infer a complete closed form expression for the Hamilton equations for the perturbations:

$$
\begin{aligned}
a_{i j}^{\prime} & =2 \gamma H^{2} a^{2} \delta e_{i j}-\gamma \epsilon_{i n m} \partial_{n} a_{m j} \\
& +\frac{1+\gamma^{2}}{\gamma} \epsilon_{i n m} \partial_{n}\left(a_{m j}-\epsilon_{m k l} \partial_{k} \delta e_{l j}\right) \\
\delta e_{i j}^{\prime} & =\frac{1}{\gamma}\left(a_{i j}-\epsilon_{i n m} \partial_{n} \delta e_{m j}\right) .
\end{aligned}
$$

Again the Hamilton equation for $\delta e_{i j}$ is equivalent to (30), and so a statement that the connection is torsion free. Combined with the Hamilton equation for $a_{i j}$ we obtain a second order equation for $\delta e_{i j}$ from which $\gamma$ drops out, as it should. This is obviously (133) again, proving consistency with cosmological perturbation theory for all $\gamma$.

By expanding the Hamiltonian to second order in first

\footnotetext{
${ }^{6}$ In a number of treatments $\Gamma$ is seen as an independent variable, which only becomes $\Gamma(E)$ on shell, and which thus commutes with $A$. This doesn't clear up the messy last term in the Hamilton equation for $A$, should we need an explicit expression.
}

order variables we obtain the counterpart of (55):

$$
\begin{aligned}
& { }_{1}^{2} \mathcal{H}=\frac{1}{2 l_{P}^{2}} \int d^{3} x\left[\frac{1}{\gamma^{2}} a_{i j} a_{i j}+2 \epsilon_{i j k} \delta e_{l i} \partial_{j} a_{k l}\right. \\
& +\frac{2}{\gamma} H a \delta e_{i j} a_{i j}-2 \frac{1+\gamma^{2}}{\gamma} H a \delta e_{i j} \epsilon_{i k l}\left(\partial_{k} \delta e_{l j}\right) \\
& -\frac{1+\gamma^{2}}{\gamma^{2}}\left[\epsilon_{i k l}\left(\partial_{k} \delta e_{l j}\right) a_{i j}+\epsilon_{i k l} a_{i j}\left(\partial_{k} \delta e_{l j}\right)\right] \\
& \left.+\frac{1+\gamma^{2}}{\gamma^{2}} \epsilon_{i k l} \epsilon_{j m n}\left(\partial_{k} \delta e_{l j}\right)\left(\partial_{m} \delta e_{n i}\right)-2 H^{2} a^{2} \delta e_{i j} \delta e_{i j}\right] .
\end{aligned}
$$

where we have been careful with the ordering, with an eye on quantization. As before the boundary term modifies the second term in the first line. The second line is cancelled by the extra term associated with the canonical transformation:

$$
\frac{\partial F}{\partial \eta}=-\frac{1}{\gamma l_{P}^{2}} \int d^{3} x H a \delta e_{i j}\left[a_{i j}-\left(1+\gamma^{2}\right) \epsilon_{i n m} \partial_{n} \delta e_{m j}\right]
$$

This can be inferred by examining the behavior of Hamilton's equations under time-dependent rescalings of $a_{i j}$ and $\delta e_{i j}$. If we try to combine them into a single second order equation, the term proportional to $\left(\gamma^{2}+1\right)$ is obviously necessary: This is because $K=(A-\Gamma) / \gamma$ contains two terms which scale differently. So in order for the time derivatives to scale in a form producing consistent equations, we need to add the second term. With these considerations the effective Hamiltonian, to be used in the second quantized theory, is therefore:

$$
\begin{aligned}
& \mathcal{H}_{e f f}=\frac{1}{2 l_{P}^{2}} \int d^{3} x\left[\frac{1}{\gamma^{2}} a_{i j} a_{i j}-2 H^{2} a^{2} \delta e_{i j} \delta e_{i j}\right. \\
& +\left(1-\frac{1}{\gamma^{2}}\right) \epsilon_{i k l}\left(\partial_{k} \delta e_{l j}\right) a_{i j}-\left(1+\frac{1}{\gamma^{2}}\right) \epsilon_{i k l} a_{i j}\left(\partial_{k} \delta e_{l j}\right) \\
& \left.+\left(1+\frac{1}{\gamma^{2}}\right) \epsilon_{i k l} \epsilon_{j m n}\left(\partial_{k} \delta e_{l j}\right)\left(\partial_{m} \delta e_{n i}\right)\right]
\end{aligned}
$$

For completeness we present the perturbative equations for the theory in terms of $\delta K_{i j}$ and $\delta e_{i j}$, i.e. in the extended ADM form, before a canonical transformation is applied [6]. This corresponds to the Palatini-Kibble limit, $|\gamma| \rightarrow \infty$, with a $S U(2)$ extension of the phase space. The equations are:

$$
\begin{aligned}
\delta K_{i j}^{\prime} & =2 H^{2} a^{2} \delta e_{i j}-\epsilon_{i n m} \partial_{n} \delta \Gamma_{m j} \\
\delta e_{i j}^{\prime} & =K_{i j} .
\end{aligned}
$$

Undoubtedly these are the simplest equations, together with $\gamma= \pm i$. It is very easy to check that these equations can be combined into an equation of the form of (133). 


\section{COMMUTATION RELATIONS IN TERMS OF MODES}

In order to quantize the theory we need to replace Poisson brackets (49) and (61) by commutators ${ }^{7}$. Thus, we obtain equal-time commutation relations:

$$
\left[A_{a}^{i}(\mathbf{x}), E_{j}^{b}(\mathbf{y})\right]=i \gamma l_{P}^{2} \delta_{a}^{b} \delta_{j}^{i} \delta(\mathbf{x}-\mathbf{y}),
$$

and:

$$
\left[a_{a}^{i}(\mathbf{x}), \delta e_{j}^{b}(\mathbf{y})\right]=-i \gamma l_{P}^{2} \delta_{a}^{b} \delta_{j}^{i} \delta(\mathbf{x}-\mathbf{y}) .
$$

These are the commutation relations before the Gauss constraint is enforced. They must be replaced by a suitably TT projected $\delta$-function after gauge fixing.

Dropping the indices for the moment, we can separate positive and negative frequencies in equation (19) as $\delta e=$ $\delta e^{+}+\delta e^{-}$where:

$$
\begin{aligned}
\delta e^{+}(\mathbf{x}, \eta) & =\int \frac{d^{3} k}{(2 \pi)^{\frac{3}{2}}} e^{+}(\mathbf{k}, \eta) e^{i \mathbf{k} \cdot \mathbf{x}} \\
\delta e^{-}(\mathbf{x}, \eta) & =\int \frac{d^{3} k}{(2 \pi)^{\frac{3}{2}}} e^{-\dagger}(\mathbf{k}, \eta) e^{-i \mathbf{k} \cdot \mathbf{x}}
\end{aligned}
$$

and likewise for $a=a^{+}+a^{-}$. The only non-vanishing equal-time commutators must be:

$$
\left[a^{+}(\mathbf{x}), \delta e^{-}(\mathbf{y})\right]=\left[a^{-}(\mathbf{x}), \delta e^{+}(\mathbf{y})\right]=-i \gamma \frac{l_{P}^{2}}{2} \delta(\mathbf{x}-\mathbf{y}),
$$

so that

$$
\left[a^{+}(\mathbf{k}), e^{-\dagger}\left(\mathbf{k}^{\prime}\right)\right]=-\left[a^{-}(\mathbf{k}), e^{+\dagger}\left(\mathbf{k}^{\prime}\right)\right]=-i \gamma \frac{l_{P}^{2}}{2} \delta\left(\mathbf{k}-\mathbf{k}^{\prime}\right),
$$

where the minus sign in the second commutator appears because $\gamma$ is imaginary. We stress that with our conventions and boundary conditions $\mathbf{k}$ and $-\mathbf{k}$ modes propagate in different directions and so they are independent. Therefore their amplitudes must commute and (77) had to be proportional to $\delta\left(\mathbf{k}-\mathbf{k}^{\prime}\right)$. This is to be contrasted with some of the literature.

Upon gauge fixing these expressions take the specific forms (19). This results in the TT-fixed commutators:

$$
\left[\tilde{a}_{r p}(\mathbf{k}), \tilde{e}_{s q}^{\dagger}\left(\mathbf{k}^{\prime}\right)\right]=-i \gamma p \frac{l_{P}^{2}}{2} \delta_{r s} \delta_{p \bar{q}} \delta\left(\mathbf{k}-\mathbf{k}^{\prime}\right),
$$

(where $\bar{q}=-q$ ). With these relations we get the expected [23] version of (73):

$$
\left[a_{i j}(\mathbf{x}), \delta e_{k l}(\mathbf{y})\right]=-i \gamma l_{P}^{2} P_{i j k l}(\mathbf{x}-\mathbf{y}),
$$

\footnotetext{
7 If the reality conditions are to be seen as second class constraints at the quantum level, we should identify the Dirac brackets at this step. We shall examine this possibility in a future article.
}

with

$$
P_{i j k l}(\mathbf{x})=\int \frac{d^{3} k}{(2 \pi)^{3}} \sum_{r} \epsilon_{i j}^{r}(\mathbf{k}) \epsilon_{k l}^{r \star}(\mathbf{k}) e^{i \mathbf{k} \cdot \mathbf{x}}
$$

(as before we mix $i$ and $a$ indices, raising and lowering them with a Kroenecker delta). Using the polarization completeness relations this can also be written as [23]:

$$
P_{i j k l}=\int \frac{d^{3} k}{(2 \pi)^{3}} \Pi_{i j k l}(\mathbf{k}) e^{i \mathbf{k} \cdot(\mathbf{x}-\mathbf{y})},
$$

with

$$
\begin{aligned}
\Pi_{i j k l}(\mathbf{k})= & \frac{1}{2}\left[\left(\delta_{i k}-\hat{k}_{i} \hat{k}_{k}\right)\left(\delta_{j l}-\hat{k}_{j} \hat{k}_{l}\right)\right. \\
& +\left(\delta_{i l}-\hat{k}_{i} \hat{k}_{l}\right)\left(\delta_{j k}-\hat{k}_{j} \hat{k}_{k}\right) \\
& \left.-\left(\delta_{i j}-\hat{k}_{i} \hat{k}_{j}\right)\left(\delta_{k l}-\hat{k}_{k} \hat{k}_{l}\right)\right]
\end{aligned}
$$

or equivalently

$$
\begin{aligned}
\Pi_{i j k l}(\mathbf{x})= & \frac{1}{2}\left[\left(\delta_{i k}-\frac{\partial_{i} \partial_{k}}{\partial^{2}}\right)\left(\delta_{j l}-\frac{\partial_{j} \partial_{l}}{\partial^{2}}\right)\right. \\
& +\left(\delta_{i l}-\frac{\partial_{i} \partial_{l}}{\partial^{2}}\right)\left(\delta_{j k}-\frac{\partial_{j} \partial_{k}}{\partial^{2}}\right) \\
& \left.-\left(\delta_{i j}-\frac{\partial_{i} \partial_{j}}{\partial^{2}}\right)\left(\delta_{k l}-\frac{\partial_{k} \partial_{l}}{\partial^{2}}\right)\right] \delta(\mathbf{x}) .
\end{aligned}
$$

We point out that (78) isn't exactly what could have been guessed, say, by quantizing a complex scalar field. The reason is that the action and Hamiltonian are not real before the reality conditions are imposed (not of the form $\left.\partial_{\mu} \phi \partial^{\mu} \phi^{\star}\right)$. Thus the classical relation between the variable $A$ and its conjugate $E$ does not involve complex conjugation (unlike in $\Pi=\dot{\phi}^{\dagger}$ ). As a result we get a $\delta_{p \bar{q}}$ in the result, i.e. non-vanishing commutators involve the negative frequencies of one variable and the positive frequencies of the other. This wouldn't happen if the Hamiltonian were manifestly real. Another oddity is the $p$ factor in the commutator: it is present only because $\gamma$ has been assumed to be imaginary.

\section{THE QUANTUM HAMILTONIAN AND A POSSIBLE REPRESENTATION FOR THE INNER PRODUCT}

We now expand the Hamiltonian found in the previous section into Fourier modes, thereby identifying the combinations of metric and connection variables to be equated with the graviton. This is a non-trivial exercise (particularly for $\gamma \neq \pm i$ ) and we invariably find twice as many particles as expected. This is because the reality conditions are yet to be imposed. At the quantum level, this is done via the choice of inner product with which the Hilbert space is endowed. Two out of the four modes are then seen to be unphysical and can be removed from the Hilbert space. These spurious modes, as it turns out, have negative energy and don't exist classically (i.e. are zero on-shell). 


\section{A. Inside the horizon, with $\gamma= \pm i$}

We consider the limit $k|\eta| \gg 1$, i.e. modes inside the horizon. We assume an $E E F$ ordering but what follows can be adapted to other orderings, with the general result explained in Section VII B. Inserting expansions (19) into (64) we find:

$$
\begin{aligned}
\mathcal{H}_{e f f} & =\frac{1}{l_{P}^{2}} \int d^{3} k \sum_{r} g_{r-}(\mathbf{k}) g_{r+}(-\mathbf{k})+g_{r-}(\mathbf{k}) g_{r-}^{\dagger}(\mathbf{k}) \\
& +g_{r+}^{\dagger}(\mathbf{k}) g_{r+}(\mathbf{k})+g_{r+}^{\dagger}(\mathbf{k}) g_{r-}^{\dagger}(-\mathbf{k}),
\end{aligned}
$$

with:

$$
\begin{aligned}
& g_{r+}(\mathbf{k})=\tilde{a}_{r+}(\mathbf{k}) \\
& g_{r+}^{\dagger}(\mathbf{k})=-\tilde{a}_{r-}^{\dagger}(\mathbf{k})+2 k r \tilde{e}_{r-}^{\dagger}(\mathbf{k}) \\
& g_{r-}(\mathbf{k})=-\tilde{a}_{r+}(\mathbf{k})+2 k r \tilde{e}_{r+}(\mathbf{k}) \\
& g_{r-}^{\dagger}(\mathbf{k})=\tilde{a}_{r-}^{\dagger}(\mathbf{k})
\end{aligned}
$$

where we used $\epsilon_{i j}^{r}(\mathbf{k}) \epsilon_{i j}^{s \star}(\mathbf{k})=2 \delta^{r s}$ (note that with our conventions $\left.\epsilon_{i j}^{r}(-\mathbf{k})=\epsilon_{i j}^{r \star}(\mathbf{k})\right)$. We have identified (anti)graviton creation and annihilation operators, $g_{r p}^{\dagger}$ and $g_{r p}$, as in [13]. From (78) they inherit the algebra:

$$
\left[g_{r p}(\mathbf{k}), g_{s q}^{\dagger}\left(\mathbf{k}^{\prime}\right)\right]=-i \gamma l_{P}^{2}(p r) k \delta_{r s} \delta_{p q} \delta\left(\mathbf{k}-\mathbf{k}^{\prime}\right) .
$$

This Hamiltonian has several strange features. For a given $\mathbf{k}$ (and here this means modes moving along $\mathbf{k}$ and not $-\mathbf{k})$ we find 4 , not 2 independent modes $(r= \pm 1$ and $p= \pm 1$ ). As in Witten's argument [15], half of these particles have negative energy (those with $i \gamma=p r$ ). For example, for the SD connection the left "graviton" $(r=-1$ and $p=1)$ and the right "anti-graviton" $(r=1$ and $p=-1$ ) carry negative energy. The Hamiltonian also contains pathological particle production terms: the first and last terms in (84). Such terms, coupling $\mathbf{k}$ and $-\mathbf{k}$ modes, are pump terms [18] representing pair production, and must be unphysical in a Minkowski brackground or for $k|\eta| \gg 1$.

Both of these pathological features are not present for classical solutions, i.e. they vanish on-shell (by imposing the solutions found in Section (II). For example, for $\gamma=i$ we have $a_{R_{-}} \approx 0$ and $a_{L+} \approx 0$. Thus the negative energy modes don't exist classically and the pump terms are identically zero. Quantum mechanically these features must be erased by removing the spurious modes present in the full Hilbert space of the second quantized theory. This is done by an appropriate choice of inner product, representing the reality conditions at the quantum level, as we now show.

Notice first that the reality conditions amount to demanding that $g_{r p}^{\dagger}$ be indeed the hermitian conjugate of $g_{r p}$. This fully fixes the inner product [4, 13, 24]. We work in a holomorphic representation for wavefunctions $\Phi$ which diagonalizes $g_{r p}^{\dagger}$, i.e.:

$$
g_{r p}^{\dagger} \Phi(z)=z_{r p} \Phi(z)
$$

( $z$ represents collectively all the $\left.z_{r p}(\mathbf{k})\right)$. Then, (89) implies:

$$
g_{r p} \Phi=-i \gamma l_{P}^{2}(p r) k \frac{\partial \Phi}{\partial z_{r p}} .
$$

With the Ansatz for the inner product:

$$
\left\langle\Phi_{1} \mid \Phi_{2}\right\rangle=\int d z d \bar{z} e^{\mu(z, \bar{z})} \bar{\Phi}_{1}(\bar{z}) \Phi_{2}(z)
$$

the formal condition $\left\langle\Phi_{1}\left|g_{r p}^{\dagger}\right| \Phi_{2}\right\rangle=\overline{\left\langle\Phi_{2}\left|g_{r p}\right| \Phi_{1}\right\rangle}$ therefore requires:

$$
\mu(z, \bar{z})=\int d \mathbf{k} \sum_{r p} \frac{p r}{i k \gamma l_{P}^{2}} z_{r p}(\mathbf{k}) \bar{z}_{r p}(\mathbf{k}),
$$

fixing $\left\langle\Phi_{1} \mid \Phi_{2}\right\rangle$. Integrating $g_{r p} \Phi_{0}=0$ leads, in this representation, to the vacuum

$$
\Phi_{0}=\langle z \mid 0\rangle=1 .
$$

Particle states are monomials in the respective variables,

$$
\Phi_{n}=\langle z \mid n\rangle \propto\left(g_{r p}^{\dagger}\right)^{n} \Psi_{0}=z_{r p}^{n} .
$$

Thus, with the inner product just derived these aren't normalizable for $i \gamma=p r$. Therefore such modes should be excluded from the physical Hilbert space, and this removes all pathologies found in the Hamiltonian. We stress that the quantum modes we have disqualified don't exist classically (see discussion after (32)). For example for $\gamma=i$ the only physical modes are $g_{R}^{p h}=g_{R+}$ and $g_{L}^{p h}=g_{L-}$.

We therefore regain the usual physical Hamiltonian, but with one major difference. For $\gamma=i$ we get

$$
\mathcal{H}_{e f f}^{p h} \approx \frac{1}{l_{P}^{2}} \int d \mathbf{k}\left(g_{L}^{p h} g_{L}^{p h^{\dagger}}+g_{R}^{p h^{\dagger}} g_{R}^{p h}\right)
$$

and so only the left handed graviton needs to be normal ordered and produces a vacuum energy. For the ASD connection only the right handed graviton produces vacuum energy. This chirality feature traces directly to the fact that the Hamiltonian is not real, a priori, as explained before.

\section{B. Inside the horizon, with $\gamma^{2} \neq-1$}

We now examine the more general case of a connection which isn't SD or ASD $(\gamma= \pm i)$. We shall only consider imaginary $\gamma$ leaving the case of a generally complex $\gamma$ for a further publication [25]. The exercise is straightforward but the algebra is cumbersome.

The Hamiltonian is now quite intricate (see Appendix III), making it difficult to identify the graviton modes. Instead it's easier to look at the classical solution (obtained from the Lagrangian formalism; see Section II) and infer combinations of $\tilde{a}_{r p}, \tilde{e}_{r p}$ and their conjugates 
equivalent to the $g_{r p}$ and $g_{r p}^{\dagger}$ listed in (85) to (88). One of these combinations should be zero on-shell, and represent the unphysical quantum mode. The other should commute with it, given commutation relations (78) or (89), for all values of $\gamma$. These requirements suffice to determine:

$$
\begin{aligned}
G_{r \mathcal{P}_{+}} & =\frac{(r-i \gamma) g_{r+}-(r+i \gamma) g_{r-}}{-2 \gamma i} \\
G_{r \mathcal{P}_{-}} & =\frac{(r+i \gamma) g_{r+}-(r-i \gamma) g_{r-}}{-2 \gamma i}
\end{aligned}
$$

We have introduced a new index $\mathcal{P}=\mathcal{P}_{+}, \mathcal{P}_{-}$to label physical and non-physical modes. The notation may look strange but it has the virtue of avoiding confusions with $p= \pm$ used for positive and negative frequency. Except for the cases of $\gamma= \pm i$ the two don't align. Thus, $\mathcal{P}=\mathcal{P}_{+}=1$ denotes the physical modes, which shouldn't vanish classically, and quantum mechanically are expected to have positive energy and norm; $\mathcal{P}=\mathcal{P}_{-}=-1$ denotes modes that classically vanish, and quantum mechanically are expected to have negative energy and norm.

It is easy to see that on-shell and enforcing the reality conditions, $G_{r \mathcal{P}_{-}} \approx 0$ and $G_{r \mathcal{P}_{+}} \approx 2 r k e_{r}$, so our first requirement is satisfied. Furthermore we find commutation relations:

$$
\begin{aligned}
{\left[G_{r \mathcal{P}}(\mathbf{k}), G_{s \mathcal{P}}^{\dagger}\left(\mathbf{k}^{\prime}\right)\right] } & =\mathcal{P} k l_{P}^{2} \delta_{r s} \delta\left(\mathbf{k}-\mathbf{k}^{\prime}\right) \\
{\left[G_{r \mathcal{P}_{+}}(\mathbf{k}), G_{s \mathcal{P}_{-}}^{\dagger}\left(\mathbf{k}^{\prime}\right)\right] } & =0
\end{aligned}
$$

as required. Notice that these combinations are not a rotation upon the original modes found for $\gamma= \pm i$. A pure rotation would leave $\left[G_{r \mathcal{P}_{+}}, G_{r \mathcal{P}_{-}}^{\dagger}\right] \neq 0$.

It is useful to write these modes directly in terms of triad and connection variables. Their general (off-shell, before imposing reality conditions) expression is:

$$
G_{r \mathcal{P}_{+}}=\frac{-r}{i \gamma}\left(\tilde{a}_{r+}-k(r+i \gamma) \tilde{e}_{r+}\right)
$$

$$
\begin{gathered}
G_{r \mathcal{P}_{+}}^{\dagger}=\frac{r}{i \gamma}\left(\tilde{a}_{r-}^{\dagger}-k(r-i \gamma) \tilde{e}_{r-}^{\dagger}\right) \\
G_{r \mathcal{P}_{-}}=\frac{-r}{i \gamma}\left(\tilde{a}_{r+}-k(r-i \gamma) \tilde{e}_{r+}\right) \\
G_{r \mathcal{P}_{-}}^{\dagger}=\frac{r}{i \gamma}\left(\tilde{a}_{r-}^{\dagger}-k(r+i \gamma) \tilde{e}_{r-}^{\dagger}\right)
\end{gathered}
$$

It is a straightforward algebraic exercise to show that inside the horizon (i.e. setting $H=0$ ) the off-shell Hamiltonian of Appendix III can be written as:

$$
\begin{aligned}
& \mathcal{H}_{e f f}=\frac{1}{2 l_{P}^{2}} \int d^{3} k \sum_{r}-(1+i \gamma r) G_{r} \mathcal{P}_{+}(\mathbf{k}) G_{r \mathcal{P}_{-}}(-\mathbf{k})-(1-i \gamma r) G_{r} \mathcal{P}_{-}(\mathbf{k}) G_{r \mathcal{P}_{+}}(-\mathbf{k}) \\
& +(1+i \gamma r) G_{r \mathcal{P}_{+}}(\mathbf{k}) G_{r \mathcal{P}_{+}}^{\dagger}(\mathbf{k})+(1-i \gamma r) G_{r \mathcal{P}_{+}}^{\dagger}(\mathbf{k}) G_{r} \mathcal{P}_{+}(\mathbf{k})+(1-i \gamma r) G_{r} \mathcal{P}_{-}(\mathbf{k}) G_{r \mathcal{P}_{-}}^{\dagger}(\mathbf{k})+(1+i \gamma r) G_{r \mathcal{P}_{-}}^{\dagger}(\mathbf{k}) G_{r} \mathcal{P}_{-}(\mathbf{k}) \\
& -(1-i \gamma r) G_{r \mathcal{P}_{+}}^{\dagger}(\mathbf{k}) G_{r}^{\dagger} \mathcal{P}_{-}(-\mathbf{k})-(1+i \gamma r) G_{r \mathcal{P}_{-}}^{\dagger}(\mathbf{k}) G_{r \mathcal{P}_{+}}^{\dagger}(-\mathbf{k})
\end{aligned}
$$

(In spite of its horrendous appearance, this is nothing but a generalization of Eqn. (84)). The Hamiltonian contains the same pathologies previously found for $\gamma= \pm i$, and again these are removed once the reality conditions are taken into account. This can be done with the choice of inner product.

As before, we work in a holomorphic representation which diagonalizes $G_{r \mathcal{P}}^{\dagger}$, i.e.:

$$
G_{r \mathcal{P}}^{\dagger} \Phi(z)=z_{r \mathcal{P}} \Phi(z)
$$

Then, (99) implies:

$$
G_{r \mathcal{P}} \Phi=\mathcal{P} k l_{P}^{2} \frac{\partial \Phi}{\partial z_{r \mathcal{P}}} .
$$

Formally nothing seems very different, but note that the variables $z_{r \mathcal{P}}$ expressed in terms of metric and connection variables are different from the $\gamma= \pm i$ case. With the same Ansatz (92) and the same formal condition $\left\langle\Phi_{1}\left|G_{r \mathcal{P}}^{\dagger}\right| \Phi_{2}\right\rangle=\overline{\left\langle\Phi_{2}\left|G_{r \mathcal{P}}\right| \Phi_{1}\right\rangle}$ we obtain:

$$
\mu(z, \bar{z})=\int d \mathbf{k} \sum_{r \mathcal{P}} \frac{-\mathcal{P}}{k l_{P}^{2}} z_{r \mathcal{P}}(\mathbf{k}) \bar{z}_{r \mathcal{P}}(\mathbf{k}),
$$

fixing the inner product. Formally, we therefore have the same vacuum

$$
\Phi_{0}=\langle z \mid 0\rangle=1
$$

but we stress again that the variables (and also the inner product) are different, so this is not an equivalent vacuum. Particle states are still monomials in the new 
variables:

$$
\Phi_{n}=\langle z \mid n\rangle \propto\left(G_{r \mathcal{P}}^{\dagger}\right)^{n} \Psi_{0}=z_{r \mathcal{P}}^{n}
$$

but now the non-normalizable states are the non-physical modes $\mathcal{P}=\mathcal{P}_{-}=-1$.

The physical Hamiltonian is therefore:

$\mathcal{H}_{\text {eff }}^{p h} \approx \frac{1}{2 l_{P}^{2}} \int d \mathbf{k} \sum_{r}\left[G_{r}^{p h} G_{r}^{p h \dagger}(1+i r \gamma)+G_{r}^{p h \dagger} G_{r}^{p h}(1-i r \gamma)\right]$

where $G_{r}^{p h}=G_{r \mathcal{P}_{+}}$. We can see that after normal ordering, right and left particles are exactly symmetric, but a chiral vacuum energy $V_{r}$ is found with:

$$
\frac{V_{R}-V_{L}}{V_{R}+V_{L}}=i \gamma
$$

Strictly speaking this calculation only covers imaginary $\gamma$. If $|\gamma|>1$, the vacuum energy of one of the modes becomes negative. This may signal underlying fermionic degrees of freedom [26]. It also implies that the calculation cannot be valid for the power spectrum, a matter on which we now comment.

\section{VACUUM ENERGY VS. FLUCTUATIONS AND ORDERING ISSUES}

We conclude with two final refinements to our calculation. Firstly, we make the important distinction between vacuum energy and vacuum fluctuations. It turns out that the chirality in these two quantities is identical when $\gamma= \pm i$, but not otherwise. Secondly we note that our results depend on the ordering prescription. We comment on this dependence and explain why it is a valuable asset.

\section{A. Vacuum energy and vacuum fluctuations}

In the standard inflationary calculation, the vacuum fluctuations (or their 2-point function) closely mimic the vacuum energy. In both cases, it is important to compute the time-dependent functions (25) multiplying creation and annihilation operators. These provide the same spectrum for vacuum energy and fluctuations, converting a $1 / k$ spectrum inside the horizon into a scale-invariant $1 / k^{3}$ spectrum for $|k \eta| \ll 1$ for a deSitter background. Strictly speaking, however, the chirality computed in Section VI refers to the vacuum energy. It turns out that the chiral asymmetry in the vacuum energy and in its fluctuations is identical for the extreme cases $\gamma= \pm i$, but not otherwise. This might have been expected from the fact that for $|\gamma|>1$ one of the helicity modes has negative vacuum energy. Its two-point function, being a variance, must however always be positive.

We want to compute the power spectrum:

$$
\left\langle 0\left|A_{r}^{\dagger}(\mathbf{k}) A_{r}\left(\mathbf{k}^{\prime}\right)\right| 0\right\rangle=P_{r}(k) \delta\left(\mathbf{k}-\mathbf{k}^{\prime}\right),
$$

where $A_{r}(\mathbf{k})$ is the Fourier component with handedness $r$ of the connection. Up to normalization factors this is given by:

$$
A_{r}(\mathbf{k})=a_{r+}(\mathbf{k}) e^{-i k \cdot x}+a_{r-}^{\dagger}(\mathbf{k}) e^{i k \cdot x}
$$

before reality conditions are imposed and the physical states selected. At this stage there isn't any chirality in the 2-point function; it only creeps in once the physical states are selected. For example, for $\gamma=i$ we find that

$$
\begin{aligned}
& A_{R}^{p h}(\mathbf{k})=a_{R+}(\mathbf{k}) e^{-i k \cdot x}=g_{R+}(\mathbf{k}) e^{-i k \cdot x} \\
& A_{L}^{p h}(\mathbf{k})=a_{L+}^{\dagger}(\mathbf{k}) e^{i k \cdot x}=g_{L+}^{\dagger}(\mathbf{k}) e^{i k \cdot x}
\end{aligned}
$$

which creates a chiral asymmetry (the right handed physical mode is represented by an annihilation operator; the left-handed by a creation operator). And indeed:

$$
\begin{aligned}
& \left\langle 0\left|A_{R}^{p h \dagger}(\mathbf{k}) A_{R}^{p h}\left(\mathbf{k}^{\prime}\right)\right| 0\right\rangle=\left\langle 0\left|g_{R+}^{\dagger}(\mathbf{k}) g_{R+}(\mathbf{k})\right| 0\right\rangle=0 \\
& \left\langle 0\left|A_{L}^{p h \dagger}(\mathbf{k}) A_{L}^{p h}\left(\mathbf{k}^{\prime}\right)\right| 0\right\rangle=\left\langle 0\left|g_{L-}(\mathbf{k}) g_{L_{-}}^{\dagger}(\mathbf{k})\right| 0\right\rangle \neq 0
\end{aligned}
$$

leading to vacuum fluctuations for left gravitons only.

For a general $\gamma$, Eqns. (101)-1102) can be used to express the positive and negative frequencies of the connection in terms of physical modes according to:

$$
\begin{aligned}
a_{r+}^{p h} & =\frac{r-i \gamma}{2 r} G_{r \mathcal{P}_{+}} \\
a_{r+}^{p h \dagger} & =\frac{r-i \gamma}{2 r} G_{r \mathcal{P}_{+}}^{\dagger} \\
a_{r-}^{p h} & =\frac{r+i \gamma}{2 r} G_{r \mathcal{P}_{+}} \\
a_{r-}^{p h \dagger} & =\frac{r+i \gamma}{2 r} G_{r \mathcal{P}_{+}}^{\dagger} .
\end{aligned}
$$

Thus when we write (113) in terms of physical modes we obtain:

$$
\begin{aligned}
A_{r}^{p h}(\mathbf{k}) & =\frac{r-i \gamma}{2 r} G_{r \mathcal{P}_{+}}(\mathbf{k}) e^{-i k \cdot x}+\frac{r+i \gamma}{2 r} G_{r \mathcal{P}_{+}}^{\dagger}(\mathbf{k}) e^{i k \cdot x} \\
A_{r}^{p h \dagger}(\mathbf{k}) & =\frac{r+i \gamma}{2 r} G_{r} \mathcal{P}_{+}(\mathbf{k}) e^{-i k \cdot x}+\frac{r-i \gamma}{2 r} G_{r \mathcal{P}_{+}}^{\dagger}(\mathbf{k}) e^{i k \cdot x}
\end{aligned}
$$

so that

$$
\left\langle 0\left|A_{r}^{p h \dagger}(\mathbf{k}) A_{r}^{p h}\left(\mathbf{k}^{\prime}\right)\right| 0\right\rangle=\frac{(r+i \gamma)^{2}}{4}\left\langle 0\left|G_{r} \mathcal{P}_{+}(\mathbf{k}) G_{r \mathcal{P}_{+}}^{\dagger}(\mathbf{k})\right| 0\right\rangle .
$$

This quantity is always positive for imaginary $\gamma$, as it should be. We can now evaluate the chiral asymmetry in the power spectrum, with the result:

$$
\frac{P_{R}-P_{L}}{P_{R}+P_{L}}=\frac{2 i \gamma}{1-\gamma^{2}}
$$

This is the counterpart to Eq. (111), the asymmetry in the vacuum energy. We see that the expressions only agree for $\gamma= \pm i$, which maximize the chirality. The chirality vanishes in the limits $|\gamma| \rightarrow 0$ and $|\gamma| \rightarrow \infty$, the latter representing the Palatini-Kibble theory. 


\section{B. Ordering and experiment in quantum mechanics}

Our results depend on the ordering prescription. This is not a weakness peculiar to our paper or indeed to quantum gravity: it is a general shortcoming of quantum mechanics. Many quantum ordering issues are ultimately decided by experiment. Failing that, there's prejudice. Arguments have been put forward in favor of " $E$ s to the right of $A \mathrm{~s}$ " (it leads to simpler expressions in the representation diagonalizing the connection), but support for the opposite ordering is now ubiquitous in the literature. For the Hamiltonian constraint these orderings translate into $F E E$ or $E E F$ ordering, but " $E F E$ " and symmetric orderings are also popular, with the $E E K K$ term (present if $\gamma \neq \pm i$ ) always symmetrized.

Theoretical arguments may be interesting and even useful, but as with any other quantum mechanical description, ultimately one must appeal to experiment to settle the matter ${ }^{8}$. It is in this spirit that we consider the dependence of our results upon ordering as an asset rather than a liability. Our results can assist us in resolving ordering issues via experiment.

Consider a quantum version of the Hamiltonian (44), with symmetric $E E K K$ term, and the following general ordering for the other term:

$$
E E F \rightarrow \alpha E E F+\beta F E E+\delta E F E
$$

with $\alpha+\beta+\delta=1$. It is easy to adapt the calculation in Section VI to find that the counterpart of (111) is now:

$$
\frac{V_{R}-V_{L}}{V_{R}+V_{L}}=i \gamma(\alpha-\beta) .
$$

The asymmetry in the 2-point function is subject to a similar ordering issue. We can replace (112) by:

$$
A^{\dagger} A \rightarrow \epsilon A^{\dagger} A+\zeta A A^{\dagger},
$$

with $\epsilon+\zeta=1$ and $\epsilon, \zeta>0$. This leads to

$$
\frac{P_{R}-P_{L}}{P_{R}+P_{L}}=\frac{2(\epsilon-\zeta) i \gamma}{1-\gamma^{2}}
$$

generalizing (121). In principle the parameters $\{\alpha, \beta, \delta\}$ can be independent of $\{\epsilon, \zeta\}$, so we have derived independent expressions. In a future publication [27], however, we shall explain how the ordering of the Hamiltonian and the two-point function are related, once one proposes a concrete wave function for the ground state of the theory.

What are the phenomenological implications of this result? For a fuller discussion we refer the reader to [28].

\footnotetext{
8 Notice that the requirement of hermiticity, much used in more "down to earth" atomic and condensed matter physics, is of no use here, since the inner product is not known a priori. Even when hermiticity can be appealed to ambiguities creep in, e.g. in atomic physics.
}

We want to stress, however, that the toy model employed there is in no way related to the calculation presented in this paper, but a translation is easy to carry out. We note that expression (125) is the only relevant input into the calculation of the TB and $\mathrm{EB}$ components of the polarization, as detailed in [28]. Specifically, adapting the calculation in [28] leads to:

$$
\frac{C_{2}^{T B}}{C_{2}^{B B}} \approx 800 \frac{(\epsilon-\zeta) i \gamma}{1-\gamma^{2}}
$$

for the ratio of tensor induced $\mathrm{TB}$ and $\mathrm{BB}$ quadrupole modes. This is an interesting quantity to consider, since it allows us to quantify how much easier the detection of a gravitational wave background (should it exist) would be rendered by chirality, regardless of the precise details of the model. The implication is that for a standard "extreme" ordering (e.g. A's to the left of E's) a TB measurement would be larger than a BB signal for an imaginary Immirzi parameter in the rough range

$$
\frac{1}{800}<|\gamma|<800 \text {. }
$$

However, any direct constraints on the Immirzi parameter from current bounds will necessarily be intertwined with parameter $r$ (the tensor to scalar ratio) and therefore be very model dependent. For example, if $r=0$, obviously no constraint can arise. Our result is therefore more useful under the prospect of a positive detection of tensor modes in general (cf. [28]).

We should not be surprised by the power of the prediction in Eq. (127): TB correlates something large with something small rather than two small quantities, as is the case with BB. Therefore even modest amounts of chirality would render TB the method of choice for detecting tensor modes.

\section{CONCLUSIONS}

In this paper we provided the details behind an earlier Letter [2], where it was claimed that a re-examination of the inflationary mechanism for producing tensor modes within Ashtekar gravity would render them chiral. Our efforts were twofold: classical and quantum. Classically we "rediscovered" standard cosmological perturbation theory within Ashtekar's formalism (Sections IIII and IV]). The exercise proved far from trivial and provided the following insights.

- The problem of time and the Hamiltonian constraint Time evolution in General Relativity is a diffeormorphism. Since the theory is diffeormorphism invariant, time evolution reduces to a constraint: the Hamiltonian constraint. This leads to the problem of time - or of the lack thereofin quantum gravity. Perturbation theory provides an insight on how time might appear as an illusion within a theory which has none. Perturbations arise in a Russian doll scenario. Expanding 
the variables into first order, second order, and so on, we find that structures, such as the Hamiltonian constraint, exhibit an inter-locked structure within the perturbative expansion (see Section IVB). For example, the second order Hamiltonian constraint ${ }^{2} \mathcal{H}$ is made up of terms quadratic in first order variables, ${ }_{1}^{2} \mathcal{H}$, and terms linear in second order variables, ${ }_{2}^{2} \mathcal{H}$. If all we care about are the first order variables (such as in cosmological perturbation theory) then only the first type of terms matter and the Hamiltonian constraint does not apply to them. The diffeormorphism invariance of full GR is then broken and we perceive a dynamical time. It is the terms linear in the second order variables, usually called the backreaction, that enforce diffeomorphism invariance to second order, but these are ignored perturbatively. Time is an illusion of perturbation theory, and yet that's the set up we live in.

- Perturbation variables as a canonical transformation If one expands the full Ashtekar variables using perturbation variables used by cosmologists and naively evaluates ${ }_{1}^{2} \mathcal{H}$, then, contrary to expectations, one does not obtain the standard cosmological Hamiltonian (see Section IV). The mystery is solved by regarding the perturbative expansions (59) and (60) as a canonical transformation into new variables, $a_{a}^{i}$ and $\delta e_{i}^{a}$, which happen to be "small" (see Section IVC). Thus the matter is far from pedantic, but it also gives a more rigorous meaning to the perturbative quantization procedure. We are not quantizing the fluctuations whilst "freezing" the quantum mechanics of the background; we are merely quantizing the full theory in new variables, which happen to be "small" in some circumstances.

- Boundary term The boundary term described by equation (46) has been often ignored in the literature, usually invoking suitable fall-off conditions [13, 21]. In SectionIV C we saw that this leads to the wrong result for the cosmological Hamiltonian. The reason is that planes waves, the central tool of cosmological perturbation theory, do not satisfy the fall-off conditions, say, in a deSitter background. Therefore the boundary term has to be included in order to obtain the correct Hamiltonian to be employed in quantizing the graviton modes (Section VI).

- Torsion and the Gauss constraint Solving for the perturbations to first order allows for a nonvanishing torsion and Gauss constraint to second order (in the form of expressions quadratic in linear perturbation variables). To use the notations defined in the text, ${ }_{1}^{2} G_{i} \neq 0$ and ${ }_{1}^{2} T^{a} \neq 0$. As with the Hamiltonian constraint, it is the second order (or backreaction) terms ${ }_{2}^{2} G_{i}$ and ${ }_{2}^{2} T^{a}$, that enforce these constraints to second order. In deriving the Ashtekar Hamiltonian from the ADM formalism 6] these two constraints are used, and therefore ${ }_{1}^{2} \mathcal{H}$ acquires extra terms. However, these turn out to be irrelevant full divergences (see Section IVB).

In the second part of the paper (Sections $\mathrm{V}$, VI and (VII) we then quantized this Hamiltonian theory. Our work corrects a number of deficiencies present in previous efforts [13, 14], and our improvements can be traced to expansions (19). These always assign the correct direction of motion and polarization to each mode, thereby removing spurious couplings between $\mathbf{k}$ and $-\mathbf{k}$ modes, as explained in Section II] The commutation relations were identified in Section $\bar{\nabla}$, where it was noted that the theory is very different from a complex scalar field, since its Hamiltonian is complex off-shell. The quantum Hamiltonian was evaluated in Section VI, and it was used to read off creation and annihilation operators for the graviton; these are chirally described in terms of metric and connection operators. In this paper we explained in detail the calculation outlined in [2], and extended it for $\gamma \neq \pm i$. In all cases we mimic Witten's result [15], initially devised for Yang-Mills theory: half the graviton modes have negative energies. However this is before reality conditions are imposed, as made evident by the fact that we have twice as many modes as needed: gravitons and anti-gravitons with right and left polarizations. Upon determination of the inner product (representing the reality conditions) we find that half of these modes are not physical and these are the modes which have negative energies in the quantum theory (and which also can be found not to satisfy the classical equations of motions). Therefore only non-pathological modes survive once the inner product and the reality conditions are taken into account. A major novelty emerges, however. We find that the physical modes have a chiral representation in terms of metric and connection operators, the exact expression depending on Immirzi parameter $\gamma$. As a result the spectrum of gravitons is unchanged, but the vacuum fluctuations and energy are chiral, with obvious cosmological consequences [28]. In Section VII we spelled out how the exact expression depends not only on $\gamma$ but also on the ordering prescription being used.

What is the physical origin of our main result? As we stressed in Section IVB the Hamiltonian of quantum gravity is intrinsically complex. It only becomes real onshell, because the Hamiltonian constraint forces it to vanish. However, when we identify the terms that drive the perturbative gravitons, ${ }_{1}^{2} \mathcal{H}$, we find that this constraint is waived, as explained in Section IVB. Then, we have to face the fact that for an imaginary (or more generally complex) $\gamma$, the Hamiltonian is complex, which is ultimately the root of all the new results reported in this paper. Many oddities found in Sections V] VI and VII can be directly traced to this fact, as highlighted in the text. We do stress, however, that we never lose Hermiticity. In fact the Hamiltonian is Hermitian with respect to the inner product used to implement the reality 
conditions (Section VI). In this respect quantum gravity is very similar to the "non-Hermitian" Hamiltonians studied by Bender and collaborators 29]. These might be regarded as nothing more than complex Hamiltonians which are in fact Hermitian with respect to a non-trivial inner product.

Whilst the considerations in the previous paragraph are an important contribution to understanding the physical origin of our results, there probably exists an underlying deeper reason. One possibility presents itself by noting that the Immirzi parameter is associated with a surface or topological term (see, for example, Eqn. (23)). This suggests that the new effects may be due to instanton fluctuations ${ }^{9}$. However this is far from obvious in our calculation, where $\gamma$ is more readily understood as a parameter related to a canonical transformation. Consequently, bridging our results with those of [32] is far from straightforward (for example, how do the graviton operators derived in Section VI fit in with the transition amplitudes of [32]?). Nonetheless this remains a very interesting conjecture and should be the subject of further investigation.

In future work [27] we hope to shed more light on our results by evaluating the wave function representing the vacuum state identified in this paper. Obviously we have derived a range of different vacua, one for each value of $\gamma$. A major result, to be presented in [27], but which we wish to highlight here, is that the vacua of the second quantized perturbation theories examined in this paper are never the perturbed Kodama state, which therefore can never describe standard gravitons 14]. The reason rests on a very simple and clear-cut algebraic fact. The implication is very deep. It appears that taking the semiclassical limit of quantum gravity is never the correct path for a perturbative, but fully quantized theory. The perturbed semi-classical limit is in fact at odds with the full quantization of the perturbations. We will expand on this fact making contact with previously published work on other vacuum states [26]. We'll also explain the relation with previous work on the graviton propagator [30], where a chiral contribution was found. The relation with our results is not obvious, since [30] employed an Euclidean signature and a real $\gamma$. However a modification of their work can be explained within our framework.

In this paper we have restricted ourselves to an imaginary Immirzi parameter. Throughout we have flagged a number of places where this assumption was used. Relaxing it renders several formulae rather cumbersome, and the algebra more involved. These changes will be presented in a future publication [25], where we'll also explain a number of further subtleties that arise if $\gamma$ is allowed to be real or have a real part. Nonetheless, the final result is, remarkably, very simple. After a lengthy calculation it turns out that for a complex $\gamma$ the chiral-

${ }^{9}$ We thank the referee for bringing this possibility to our attention. ity formulae presented in this paper are trivially changed by replacing $\gamma$ by its imaginary part. An immediate implication is that according to our calculations the real theory has no chirality at all. A full discussion of the implications will be presented in [25].

On a more cosmological front, another issue we'd like to revisit is the behavior of the modes outside the horizon. Inspection of the Hamiltonian reveals that the dynamics then becomes fully driven by the metric, with the connection pushed aside. Thus, whereas the Ashtekar formalism may be expected to result in novelties inside the horizon, a complete reduction to the second order formalism is expected as the modes leave the horizon. The problem of decoherence in cosmology may benefit from this insight. It seems that at the same time as the quantum modes lose their phase and prepare themselves to "go classical", the quantum distinction between metric and connection also evaporates.

In the meantime we have shown how a perturbative re-examination of quantum gravity can be fruitful. We hope to have cleared up a few misconceptions and paradoxes. Above all, we derived a striking prediction for the theory, which could be tested in upcoming CMB polarization experiments. There are other mechanisms to generate gravitational chirality (e.g. 26, 31, 32]), but the one pointed out in this paper is by far the simplest. As stressed in [28], even modest chirality in the gravitational wave background would render its detection far easier, and probably within the reach of the PLANCK mission.

\section{Acknowledgments}

We thank Martin Bojowald, Gianluca Calcagni, Carl Bender, Jonathan Halliwell, Chris Isham, Kirill Krasnov, Hermann Nicolai, Lee Smolin, Julian Sonner, Kelly Stelle and Thomas Thiemann for help regarding this project.

\section{Appendix I: A precis of cosmological perturbation theory}

Here we briefly collect the relevant results of cosmological tensor perturbation theory (see for example [7, 8] ). As in the main text we start from:

$$
d s^{2}=a^{2}\left(-d \eta^{2}+\left(\delta_{i j}+h_{i j}\right) d x^{i} d x^{j}\right)
$$

where $h_{i j}$ is divergenceless and tracefree. Then (raising an lowering the indices of $h_{i j}$ with $\delta_{i j}$ ), the second order Einstein-Hilbert action is:

$$
S=\frac{1}{64 \pi G} \int a^{2}\left(h^{\prime i j} h_{i j}^{\prime}-h^{i j, k} h_{i j, k}\right) d^{3} x d \eta
$$

where ' denotes derivative with respect to $\eta$. Expanding in Fourier modes, and writing $h_{i j}(\mathbf{k}, \eta)=h(\mathbf{k}, \eta) \epsilon_{i j}(\mathbf{k})$ (where $\epsilon_{i j}$ is the polarization tensor) we can then define 
a " $v$ " variable (similar to that used for treating scalar fluctuations) by:

$$
v(\mathbf{k}, \eta)=\sqrt{\frac{\epsilon^{i j} \bar{\epsilon}_{i j}}{32 \pi G}} a h(\mathbf{k}, \eta)
$$

so that the action becomes:

$$
S=\frac{1}{2} \int d^{3} k d \eta\left(v^{\prime 2}-\left(k^{2}-\frac{a^{\prime \prime}}{a}\right) v\right) .
$$

This is identical to the action used in the treatment of scalar fluctuations. Its associated Hamiltonian is:

$$
H=\frac{1}{2} \int d^{3} k\left(v^{\prime 2}+\left(k^{2}-\frac{a^{\prime \prime}}{a}\right) v\right) .
$$

In a deSitter universe it leads to the equation of motion:

$$
v^{\prime \prime}+\left(k^{2}-\frac{2}{\eta^{2}}\right) v=0 .
$$

This can be generally solved with Bessel functions, but for deSitter the solution is very simple.

We should impose the boundary condition for $k|\eta| \gg$ 1:

$$
v \rightarrow \frac{e^{-i k \eta}}{\sqrt{2 k}} b
$$

so that upon second quantization amplitudes $b$ and $b^{\dagger}$ become annihilation and creation operators. The vacuum expectation value can then be evaluated and followed for modes outside the horizon $(k|\eta| \ll 1)$, obtaining scaleinvariance. The full solution for deSitter is:

$$
v=\frac{e^{-i k \eta}}{\sqrt{2 k}}\left(1-\frac{i}{k \eta}\right) b
$$

so that in the limit $k \eta \ll 1$ we have

$$
\left\langle\left. 0|| v(k)\right|^{2} \mid 0\right\rangle=\frac{1}{2^{\frac{3}{2}} k^{3} \eta} .
$$

The reason variable $v$ is chosen instead of $h_{i j}$ is to get rid of the friction term in

$$
h_{i j}^{\prime \prime}+2 \frac{a^{\prime}}{a} h_{i j}^{\prime}+k^{2} h_{i j}=0 .
$$

\section{Appendix II: Derivation of the generating function}

Following [22], the transformation between two sets of canonical variables is achieved by a generating function that links the different variables. If the canonical transformation is time dependent, the Hamiltonian will change. In our case, we have a generating function $F\left(A_{a}^{i}, \delta e_{i}^{a}, \eta\right)$ that can be can be determined from

$$
\begin{aligned}
\frac{\delta F}{\delta A_{a}^{i}} & =\frac{1}{\gamma l_{P}^{2}} E_{i}^{a} \\
\frac{\delta F}{\delta\left(\delta e_{i}^{a}\right)} & =-\frac{1}{\gamma l_{P}^{2}} a_{a}^{i}
\end{aligned}
$$

The new Hamiltonian $\mathcal{K}$ is related to the old one by

$$
\mathcal{K}\left(a_{a}^{i}, \delta e_{i}^{a}\right)=\mathcal{H}\left(a_{a}^{i}, \delta e_{i}^{a}\right)+\frac{\partial F}{\partial \eta}\left(a_{a}^{i}, \delta e_{i}^{a}\right)
$$

where $\mathcal{H}$ and $\frac{\partial F}{\partial \eta}$ have to be expressed in terms of the new variables. Solving equations (138) and (139) yields

$$
F\left(A_{a}^{i}, \delta e_{i}^{a}, \eta\right)=\int d^{3} x \frac{1}{\gamma l_{P}^{2}}\left(a^{2} A_{i}^{i}-a \delta e_{i}^{a} A_{a}^{i}\right)
$$

Thus,

$$
\frac{\partial F}{\partial \eta}=\int d^{3} x \frac{1}{\gamma l_{P}^{2}}\left(2 a a^{\prime} A_{i}^{i}-a^{\prime} \delta e_{i}^{a} A_{a}^{i}\right)
$$

Expressing this in terms of the new variables (and ignoring a zeroth order contribution) we find

$$
\frac{\partial F}{\partial \eta}\left(a_{a}^{i}, \delta e_{i}^{a}, \eta\right)=\int d^{3} x \frac{-1}{\gamma l_{P}^{2}} H a \delta e_{i}^{a} a_{a}^{i}
$$

Using equation (140), the corrected Hamiltonian is therefore

$$
\begin{aligned}
\mathcal{H}_{e f f}= & \frac{1}{2 l_{P}^{2}} \int d^{3} x\left[-a_{i j} a_{i j}-2 \epsilon_{i j k}\left(\partial_{j} \delta e_{l i}\right) a_{k l}\right. \\
& \left.-2\left(\gamma+\frac{1}{\gamma}\right) H a \delta e_{i j} a_{i j}-\left(\gamma^{2}+3\right) H^{2} a^{2} \delta e_{i j} \delta e_{i j}\right] .
\end{aligned}
$$

\section{Appendix III: General form of the off-shell Hamiltonian in terms of metric and connection variables}

For general $\gamma$, the Hamiltonian in terms of Fourier modes can be found by substituting the expansions (19) into the perturbative Hamiltonian (69): 


$$
\begin{aligned}
\mathcal{H}_{e f f}= & \frac{1}{l_{P}^{2}} \int d^{3} k \sum_{r} \frac{1}{\gamma^{2}}\{ \\
& {\left[\left\{k^{2}\left(\gamma^{2}+1\right)-2 \gamma^{2} H^{2} a^{2}\right\} \tilde{e}_{r+}(\mathbf{k})-k r\left(\gamma^{2}+1\right) \tilde{a}_{r+}(\mathbf{k})\right] \tilde{e}_{r+}(-\mathbf{k}) } \\
+ & {\left[\left\{k^{2}\left(\gamma^{2}+1\right)-2 \gamma^{2} H^{2} a^{2}\right\} \tilde{e}_{r+}(\mathbf{k})-k r\left(\gamma^{2}+1\right) \tilde{a}_{r+}(\mathbf{k})\right] \tilde{e}_{r-}^{\dagger}(\mathbf{k}) } \\
+ & {\left[\left\{k^{2}\left(\gamma^{2}+1\right)-2 \gamma^{2} H^{2} a^{2}\right\} \tilde{e}_{r-}^{\dagger}(\mathbf{k})-k r\left(\gamma^{2}+1\right) \tilde{a}_{r-}^{\dagger}(\mathbf{k})\right] \tilde{e}_{r+}(\mathbf{k}) } \\
+ & {\left[\left\{k^{2}\left(\gamma^{2}+1\right)-2 \gamma^{2} H^{2} a^{2}\right\} \tilde{e}_{r-}^{\dagger}(\mathbf{k})-k r\left(\gamma^{2}+1\right) \tilde{a}_{r-}^{\dagger}(\mathbf{k})\right] \tilde{e}_{r-}^{\dagger}(-\mathbf{k}) } \\
+ & {\left[k r\left(\gamma^{2}-1\right) \tilde{e}_{r+}(\mathbf{k})+\tilde{a}_{r+}(\mathbf{k})\right] \tilde{a}_{r+}(-\mathbf{k}) } \\
+ & {\left[k r\left(\gamma^{2}-1\right) \tilde{e}_{r+}(\mathbf{k})+\tilde{a}_{r+}(\mathbf{k})\right] \tilde{a}_{r-}^{\dagger}(\mathbf{k}) } \\
+ & {\left[k r\left(\gamma^{2}-1\right) \tilde{e}_{r-}^{\dagger}(\mathbf{k})+\tilde{a}_{r-}^{\dagger}(\mathbf{k})\right] \tilde{a}_{r+}(\mathbf{k}) } \\
+ & {\left.\left[k r\left(\gamma^{2}-1\right) \tilde{e}_{r-}^{\dagger}(\mathbf{k})+\tilde{a}_{r-}^{\dagger}(\mathbf{k})\right] \tilde{a}_{r-}^{\dagger}(-\mathbf{k})\right\} }
\end{aligned}
$$

[1] A. Guth, Phys.Rev. D23 347 (1981); A. Linde, Phys. Lett B 108, 1220 (1982), A. Albrecht and P. Steinhardt, Phys. Rev. Lett. 48: 1220-1223, 1982.

[2] J. Magueijo and D. Benincasa, Phys. Rev. Lett. 106: 121302, 2011.

[3] A. Ashtekar, New perspectives in canonical gravity, Humanities Press Inter, 1988; A. Ashtekar and R. S. Tate, Lectures on non-perturbative canonical gravity, World Scientific 1991.

[4] R. Gambini and J. Pullin, Loops, Knots, Gauge theories and Quantum gravity, CUP, Cambridge 1996.

[5] C. Rovelli, Quantum Gravity, CUP, Cambridge, 2004.

[6] T. Thiemann, Modern Canonical Quantum General Relativity, CUP, Cambridge, 2007.

[7] A. Liddle and D. Lyth, "Cosmological Inflation and Large-scale Structure", CUP, Cambridge 2000.

[8] V. Mukhanov, "Physical Foundations of cosmology", CUP, Cambridge 2005.

[9] R. Arnowitt, S. Deser and C. W. Misner, Phys. Rev. 117, 1595, 1960.

[10] D. Langlois, Class. Quantum Grav. I1 (1994) 389407.

[11] M. Bojowald, Living Rev.Rel. 11: 4,2008;

[12] A. Ashtekar, J. Math.Phys. 27, 824, 1986.

[13] A. Ashtekar, C. Rovelli and L. Smolin, Phys. Rev. D44, 1740, 1991.

[14] L. Freidel and L. Smolin, Class. Quant. Grav. 21: 38313844, 2004.

[15] E. Witten, "A Note on the Chern-Simons and Kodama wave functions", gr-qc/0306083
[16] H. Kodama, Phys. Rev. D42, 2548, 1990.

[17] C. Misner, K. Thorner and J. Wheeler, Gravitation, W. H. Freeman and Co., New York, 1973.

[18] L. P. Grishchuk and Y. V. Sidorov, Phys. Rev. D42, 3413, 1990; L. P. Grishchuk, arXiv:0707.3319

[19] G. W. Gibbons and S. W. Hawking, Phys. Rev. D15, 2752, 1977.

[20] T. Regge and C. Teitelboim, Ann. Phys. 88, 236, 1974.

[21] A. Ashtekar, J. Eagle and D. Sloan, arXiv:0802.2527v2

[22] H. Goldstein, "Classical mechanics", Addison-Wesley, Boston, 1980.

[23] S. Weinberg, Phys. Rev. 138, B988, 1965.

[24] A. Ashtekar and R. S. Tate, J. Math. Phys. 35, 6434, 1994.

[25] L. Bethke and J. Magueijo, to be submitted.

[26] S. Alexander and G. Calcagni, Found.Phys.38, 11481184, 2008; Physics Letters B 672 (2009) 386.

[27] D. Benincasa, L. Bethke and J. Magueijo, in preparation.

[28] C. Contaldi, J. Magueijo and L. Smolin, Phys.Rev.Lett. 101: 141101, 2008.

[29] C. M. Bender, Rep. Prog. Phys. 70, 947, 2007.

[30] C. Rovelli, Phys. Rev. Lett. 97, 151301, 2006; E. Bianchi et al, Class. Quant. Grav. 23, 6989, 2006; E. Bianchi, E. Magliaro, C.Perini, Nuc. Phys. B 822, 245, 2009.

[31] S. Alexander, arXiv:0706.4481,

[32] S. Mercuri, arXiv:1007.3732.

[33] C. Rovelli, arXiv:1004.1780 and 1010.1939. 